1997 Particle Accelerator Conference, 5/12/97-5/16/97, Vancouver/BC Canada*

$$
\text { CONF- } 970503--79 \text { BNL }-64466
$$

\title{
Emittance Studies of the BNL/SLAC/UCLA 1.6 cell Photocathode rf Gun*
}

\author{
D. T. Palmer ${ }^{*}$, X. J. Wang ${ }^{\dagger}$, R. H. Miller ${ }^{*}$ M. Babzien ${ }^{\dagger}$, I. Ben-Zvi ${ }^{\dagger}$, C. Pellegrini**, J. Sheehan ${ }^{\dagger}$, \\ J. Skaritka ${ }^{\dagger}$, H. Winick*, M. Woodle ${ }^{\dagger}$, V. Yakimenko ${ }^{\dagger}$ \\ *Stanford Linear Accelerator Center, Stanford University, Stanford, CA 94309 \\ †Brookhaven National Laboratory, Accelerator Test Facility, Upton, NY 11973 \\ **University of California Los Angeles, Department of Physics, Los Angeles, CA 90095
}

\begin{abstract}
The symmetrized 1.6 cell S-band photocathode gun developed by the BNL/SLAC/UCLA collaboration is in operation at the Brookhaven Accelerator Test Facility(ATF). A novel emittance compensation solenoid magnet has also been designed, built and is in operation at the ATF. These two subsystems form an emittance compensated photoinjector used for beam dynamics, advanced acceleration and free electron laser experiments at the ATF. The highest acceleration field achieved on the copper cathode is $150 \frac{\mathrm{MV}}{\mathrm{m}}$, and the guns normal operating field is $130 \frac{\mathrm{MV}}{\mathrm{m}}$. The maximum if pulse length is $3 \mu \mathrm{s}$. The transverse emittance of the photoelectron beam were measured for various injection parameters. The $1 \mathrm{nC}$ emittance results are presented along with electron bunch length measurements that indicated that at above the $400 \mathrm{pC}$, space charge bunch lengthening is occurring. The thermal emittance, $\epsilon_{o}$, of the copper cathode has been measured.
\end{abstract}

\section{INTRODUCTION}

The BNL/SLACUCLA S-band emittance compensated [1] photoinjector has been installed at the Brookhaven Accelerator Test Facility(ATF) as the electron source for beam dynamics studies, laser acceleration and free electron laser experiments. The 1.6 cell $\mathrm{rf}$ gun is powered by a single XK5 klystron, and is equipped with a single emittance compensation solenoidal magnet. There is a short drift space between the photoinjector and the input to the first of two SLAC three meter travelling wave accelerating sections. This low energy drift space contains a copper mirror that can be used in either transition radiation studies or laser alignment. There is also a beam profile monitor/Faraday plate located $66.4 \mathrm{~cm}$ from the cathode plane. The photoinjector beam line layout is presented in figure 1 .

The high energy beam transport system consists of nine quadrupole magnets, an energy spectrometer, an energy selection slit and a high-energy Faraday cup. Diagnostics located in the high energy transport consist of beam profile monitors and strip lines. The strip lines are used for an on line laser/rf phase stability monitor.

The drive laser is a Nd:YAG master oscillator/power amplifier system. A diode pumped oscillator mode locked at $81.6 \mathrm{MHz}$ produces 21 psec FWHM pulses at 100 $\mathrm{mW}$ of average power. Gated pulses seed two flash lamp

\footnotetext{
- Work supported by the Department of Energy, contracts DE-AC0376SF00515, DE-AC02-76CH00016 and DE-FG03-92ER40793
}

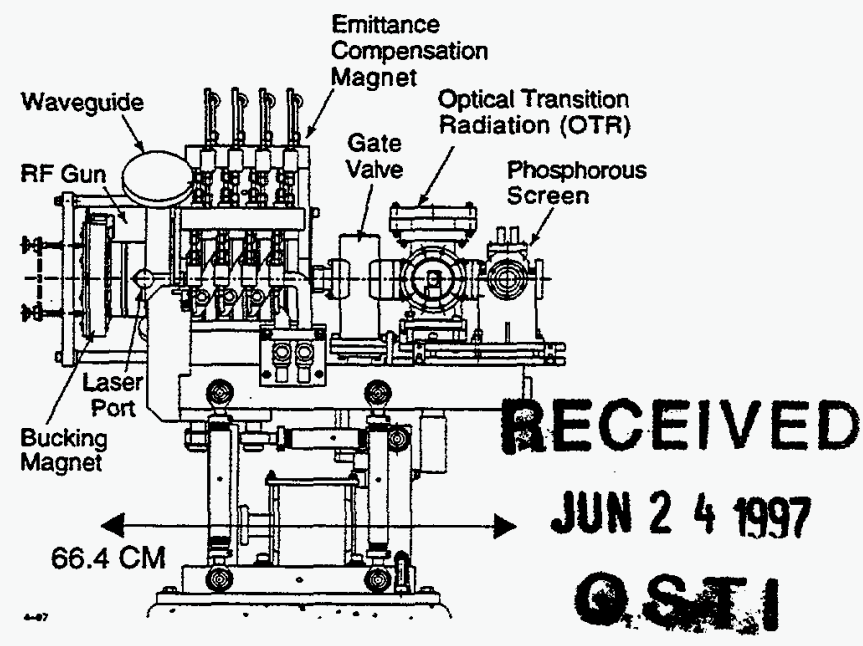

Figure 1: The BNL/UCLA/UCLA 1.6 cell S-band photoinjector

\begin{tabular}{|l|l|}
\hline Laser Spet Size & $1 \mathrm{~mm}$ radius hard edge \\
\hline Cathode Field & $130 \frac{\mathrm{MV}}{\mathrm{m}}$ \\
\hline rf Gun Beam Energy & $5.75 \mathrm{MeV}$ \\
\hline Final Beam Energy & $40 \mathrm{MeV}$ \\
\hline Cathode Magnetic Field & $\leq 5 \mathrm{G}$ \\
\hline
\end{tabular}

Table 1: Beam Study Parameters

pumped multi-pass amplifiers and are subsequently frequency quadrupled. This nonlinear process leads to a factor of two reduction in the laser pulse length. The 266 $\mathrm{nm}$ beam is transported to the rf gun area via a 20 meter long evacuated pipe. A spherical lens and a pair of Littrow prisms are used to compensate for the anamorphic magnification introduced by the $72^{\circ}$ incidence on the cathode. The time slew across the cathode caused by this oblique incidence is also corrected by using a diffraction grating. The spot size of the laser beam on the cathode is $2 \mathrm{~mm}$ diameter edge to edge.

\section{EMITTANCE STUDIES}

The normalized transverse rms emittance, $\epsilon_{\mathrm{n}, \mathrm{rms}}$, was measured using the two screen method. The measured $\epsilon_{\mathrm{n}, \mathrm{rms}}$ values that are reported are greater than or equal to the real beam emittance. Table 1 list the relevant beam parameters used during these beam dynamics studies. 


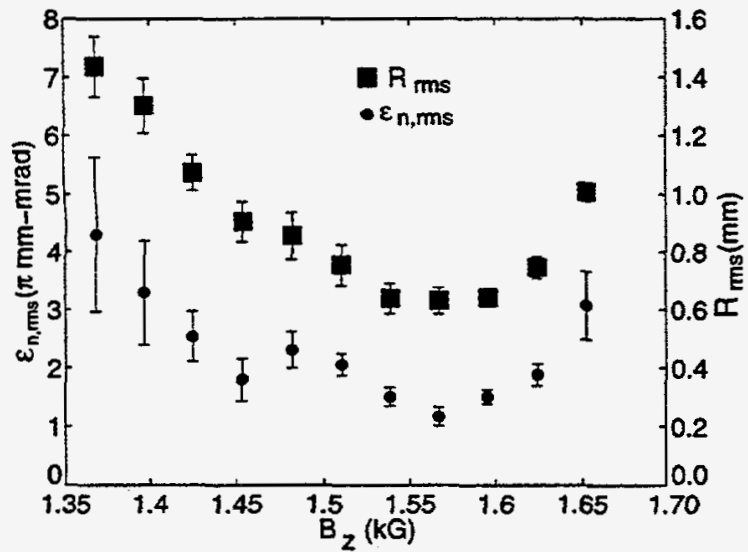

Figure 2: $\epsilon_{\mathrm{n}, \mathrm{rms}}$ and $\mathrm{R}_{\mathrm{rms}}$ versus $\mathrm{B}_{2, \max }$

\subsection{Low Charge}

PARMELA [2] was used to simulate the emittance compensation process and the subsequent acceleration to 40 $\mathrm{MeV}$. A correlation of the minimum spot size with an emittance minimum was noted during these simulations. This was experimentally verified during the commissioning of the 1.6 cell rf gun, using the beam profile monitor located at the output of the second linac section, as can be seen in figure 2 . The result in figure 2 is for a total bunch charge of $0.329 \pm 0.012 \mathrm{nC}$, an electron bunch length of $\tau_{95 \%}=$ $10.9 \mathrm{psec}$ with an $\epsilon_{\mathrm{n}, \mathrm{rms}}=1.17 \pm 0.16 \pi \mathrm{mm} \mathrm{mrad}$.

The dependence of transverse emittance on the bunch charge under two different experimental conditions are presented in figure 3 . The linear dependent emittance versus charge was conducted under constant solenoidal magnet field that was optimized for a total charge of $390 \mathrm{pC}$ and only the laser energy on the cathode was varied. For the quadratic dependent emittance versus charge, as the laser energy was varied the beam was optimized using the solenoidal magnet and steering magnets to produce the smallest symmetric beam profile at the first high energy profile screen. Note that even under these diverse experimental condition the measured emittance are consistent with the data in figure 2.

We have noted that at $Q=0$ for the linear dependence emittance versus charge there is a residual emittance term of $0.8 \pi \mathrm{mm}$-mrad. This term is due to $\epsilon_{\mathrm{o}}, \epsilon_{\mathrm{rf}}$ and $\epsilon_{\mathrm{mag}}$. The magnetic term can be neglected since the initial cathode spot size and the small magnetic field at the cathode will contribute only $0.03 \pi \mathrm{mm}$ mrad [3]. The rf contribution should be small, since there is no transverse space charge forces to cause beam size expansions in the If gun. The beam, in this limiting charge case, will only sample a small portions of the if fields which decrease the if contribution by $\sigma_{x}^{2}$ [4]. The beams intrinsic thermal emittance including the Schottky correction is given in equation 1. A theoretical estimate of the thermal emittance at $130 \frac{\mathrm{MV}}{\mathrm{m}}$ for a copper cathode was found to be $0.5 \pi \mathrm{mm}$-mrad.

$$
\epsilon_{0} \leq 0.8 \pi \mathrm{mm} \mathrm{mrad}
$$

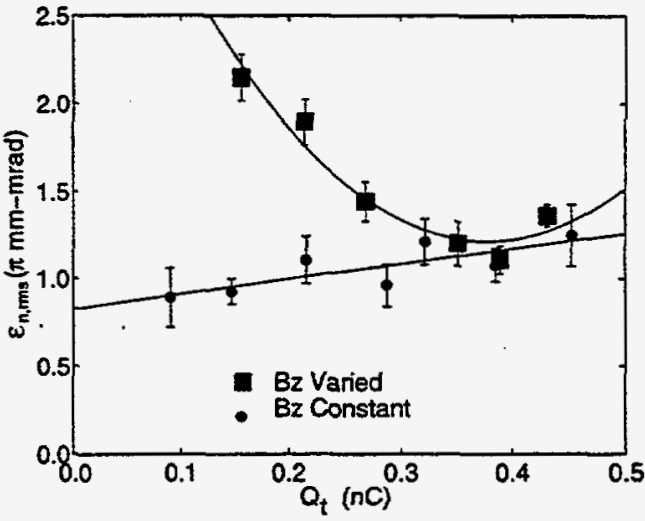

Figure 3: $\epsilon_{\mathrm{n}, \mathrm{rms}}$ versus $Q_{\mathrm{t}}$

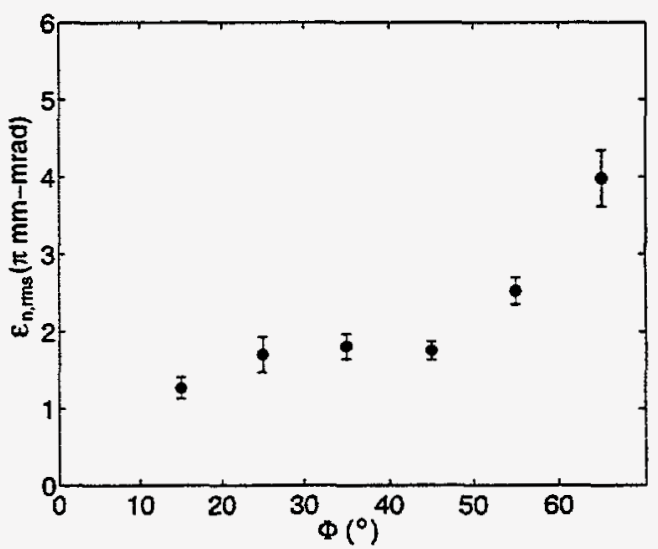

Figure 4: $\epsilon_{\mathrm{n}, \mathrm{rms}}$ versus $\phi$

Due to laser power limitation, if gun bunch compression and the Schottky effect it was not possible to keep the total charge constant for different laser injection phases. Therefore in figure 4 the plot of $\epsilon_{\mathrm{n}, \mathrm{rms}}$ as a function of laser injection phase is not for a constant charge but for a decreasing charge from a maximum of $400 \mathrm{pC}$ to a minimum of 178 pC.

\subsection{InC Emittance Studies}

For X-ray free electron laser application such as the Linear Coherent Light Source(LCLS) [5] the relevant photoinjector parameters are $1 \mathrm{nC}, \tau_{95 \%}=10 \mathrm{psec}$ and $1.00 \pi \mathrm{mm}$ mrad normalized rms emittance. The measured and LCLS values are presented in table 2 .

Simulation results using PARMELA with a longitudinal flat top electron pulse of $\tau_{95 \%}=10$ psec, full width tenth

\begin{tabular}{|l|l|l|}
\hline Parameter & LCLS & Experimental Results \\
\hline $\mathrm{Q}_{T}$ & $1 \mathrm{nC}$ & $1.020 \pm 0.059 \mathrm{nC}$ \\
\hline$\tau_{95 \%}$ & $10 \mathrm{psec}$ & $14.7 \mathrm{psec}$ \\
\hline$\epsilon_{\mathrm{n}, \mathrm{rms}}$ & $1.00 \pi \mathrm{mm} \mathrm{mrad}$ & $4.74 \pm 0.24 \pi \mathrm{mm} \mathrm{mrad}$ \\
\hline
\end{tabular}

Table 2: $1 \mathrm{nC}$ emittance results 


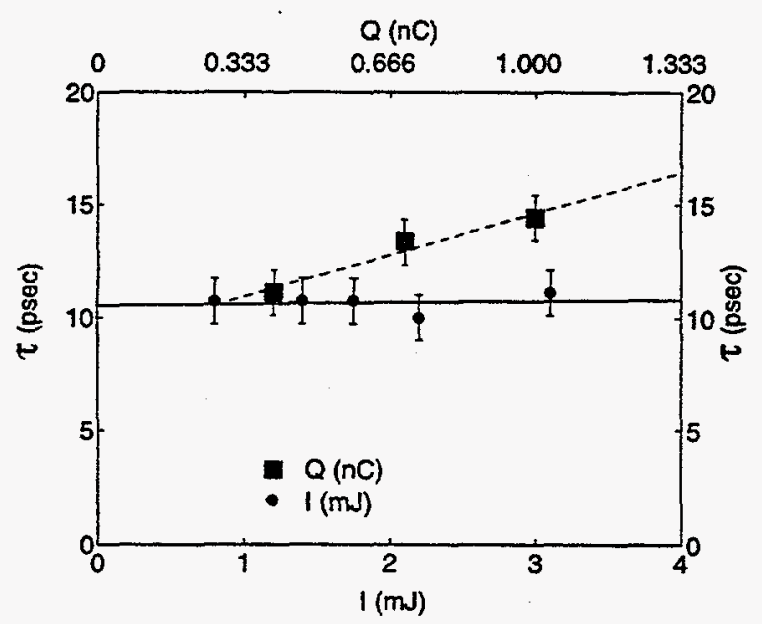

Figure 5: $\tau_{95 \%}$ versus $I_{g r e e n}$ with $Q_{t}$ constant. $\tau_{95} \%$ versus $Q_{t}$ with $I_{\text {green }}$ constant.

maximum (FWTM), indicate that a $\epsilon_{\mathrm{n}, \mathrm{rms}} \leq 1 \pi \mathrm{mm} \mathrm{mrad}$ is attainable [6].

The results of our experimental studies present two challenges. First is the increased $\tau_{95 \%}$ and second is the larger than expected $\epsilon_{\mathrm{n}, \mathrm{rms}}$. These are not unrelated since the emittance contribution due to $\mathrm{rf}$ scales as $\sigma_{\mathrm{z}}^{2}$. Therefore an important issue is to find the mechanism causing the electron bunch length to increase. Two possible mechanisms were studied; laser pulse bunch lengthening due to laser power saturation in a doubling crystal, or space charge bunch lengthening. Experimental studies of the correlation of the laser power and $\tau_{95 \%}$ were undertaken. Figure 5 shows the results of these studies. While keeping the total electron bunch charge constant at $360 \mathrm{pC}$, the laser intensity in the green $(\lambda=532 \mathrm{~nm})$ was increased. The results is that $\tau_{95 \%}=10.9$ psec. Keeping the laser intensity in the green constant and varying the uv on the cathode to increase the electron bunch charge from $400 \mathrm{pC}$ to $1 \mathrm{nC}$ results in an increase in the $\tau_{95 \%}$ from $10.9 \mathrm{psec}$ to 14.7 psec(figure 5). This clearly shows that the $1 \mathrm{nC}$ electron bunch length is not due to the laser pulse length but due to some beam dynamics issue, almost surely longitudinal space charge forces.

PARMELA simulation do not show the space charge bunch lengthening seen in these experiments and this simulation error is being investigated. This debunching effect due to space charge forces can be corrected by decreasing the laser pulse length and using the debunching effect to lengthen the $\tau_{95 \%}$ to the required $10 \mathrm{psec}$. This technique has the drawback the longitudinal phase mixing of the electron pulse could possible occur thereby degrading the emittance compensation process. A second possibility is to increase the laser spot size. But this would increase the emittance due to the if emittance contribution, the remnant magnetic field at the cathode and also increase the intrinsic thermal emittance. Using a longitudinally flat top laser pulse would seem to be the best solution since the magnetic and thermal emittance would not increase and the longitu-

\begin{tabular}{|l|l|}
\hline \multicolumn{2}{|c|}{ Before Laser Cleaning } \\
\hline$Q_{t}$ & $918 \pm 77.5 \mathrm{pC}$ \\
\hline$\epsilon_{\mathrm{n}, \mathrm{rms}}$ & $5.18 \pm 0.25 \pi \mathrm{mm}$ mrad \\
\hline \multicolumn{2}{|c|}{ After Laser Cleaning } \\
\hline$Q_{t}$ & $1020 \pm 58.7 \mathrm{pC}$ \\
\hline$\epsilon_{\mathrm{n}, \mathrm{rms}}$ & $4.74 \pm 0.24 \pi \mathrm{mm}$ mrad \\
\hline
\end{tabular}

Table 3: High charge emittance study results before and after LAEEE

dinal space charge forces would be decreased in the central portion of the bunch.

Due to the non-uniformity of the transverse laser pulse and transverse variation of the cathode quantum efficiency, the electron bunch transverse charge density $\rho(x, y)$ is not uniform. Laser assisted explosive electron emission(LAEEE) [7] has been used to smooth out this effect. The measured improvement in $\epsilon_{\mathrm{n}, \mathrm{rms}}$ due to LAEEE are presented in table 3. Qualitative data for $\mathrm{QE}(\mathrm{x}, \mathrm{y})$ of the copper cathode is not available, but for the magnesium cathode LASEEE improved the $\mathrm{QE}(\mathrm{x}, \mathrm{y})$ by a factor of two, from an order of magnitude variation across the cathode spot to a $50 \%$ after LASEEE.

\section{CONCLUSION}

We have presented the results of emittance studies of the BNL/SLAC/UCLA 1.6 cell S-band emittance compensated photoinjector:- Emittance as a function of laser injection phase, charge and peak magnetic field have been presented in the low charge regime. Emittance results for the $1 \mathrm{nC}$ regime are presented before and after laser assisted explosive electron emission cleaning. Longitudinal bunch lengthening has been observed in the $1 \mathrm{nC}$ studies and proposed solutions have been presented to counteract this effect. We have measured an upper limit to the beams intrinsic thermal emittance and found it to be $\epsilon_{\mathrm{o}} \leq 0.8 \pi \mathrm{mm}$ mrad

\section{ACKNOWLEDGMENT}

The authors would like to thank the technical staff at BNL, SLAC and UCLA for all their dedicated work on this project.

\section{REFERENCES}

[1] B. E. Carlsten, NIM, A285, 313 (1989)

[2] L. M. Young, private communications

[3] D. T. Palmer et al., Proc. 1997 Part. Accel. Conf. (1997)

[4] K. J. Kim, NIM, A275, 201 (1989)

[5] H. Winick, Electron Spectrosc. Relat. Phenom., 75, $1-8$ (1995)

[6] D. T. Palmer et al., Proc. 1995 Part. Accel. Conf. (1995) p. 2432

[7] X. J. Wang et al., J. Appl. Phys. 72(3), 888-894 


\section{DISCLAIMER}

This report was prepared as an account of work sponsored by an agency of the United States Government. Neither the United States Government nor any agency thereof, nor any of their employees, makes any warranty, express or implied, or assumes any legal liability or responsibility for the accuracy, completeness, or usefulness of any information, apparatus, product, or process disclosed, or represents that its use would not infringe privately owned rights. Reference herein to any specific commercial product, process, or service by trade name, trademark, manufacturer, or otherwise does not necessarily constitute or imply its endorsement, recommendation, or favoring by the United States Government or any agency thereof. The views and opinions of authors expressed herein do not necessarily state or reflect those of the United States Government or any agency thereof. 
DISCLAIMER

Portions of this document may be illegible in electronic image products. Images are produced from the best available original document. 